\title{
Microscopic Mechanism of Specific Peptide Adhesion to Semiconductor Substrates**
}

\author{
Michael Bachmann, Karsten Goede, ${ }^{*}$ Annette G. Beck-Sickinger, Marius Grundmann, Anders Irbäck, \\ Wolfhard Janke \\ E-mail: goede@physik.uni-leipzig.de
}

* Dr. M. Bachmann, Institut für Festkörperforschung, Theorie II, Forschungszentrum Jülich, 52425 Jülich, Germany; and Institut für Theoretische Physik, Universität Leipzig, Postfach 100920 , 04009 Leipzig, Germany; and Computational Biology \& Biological Physics, Department of Astronomy and Theoretical Physics, Lund University, Sölvegatan 14A, 22362 Lund, Sweden;

* Dr. K. Goede, Institut für Experimentelle Physik II, Universität Leipzig, Linnéstraße 5, 04103 Leipzig, Germany, goede @ physik.uni-leipzig.de, phone: +49 341 9732626, fax: +49 341 9732668;

* Prof. A. G. Beck-Sickinger, Institut für Biochemie, Universität Leipzig, Brüderstraße 34, 04103 Leipzig, Germany;

* Prof. M. Grundmann, Institut für Experimentelle Physik II, Universität Leipzig, Linnéstraße 5, 04103 Leipzig, Germany;

* Prof. A. Irbäck, Computational Biology \& Biological Physics, Department of Astronomy and Theoretical Physics, Lund University, Sölvegatan 14A, 22362 Lund, Sweden;

* Prof. W. Janke, Institut für Theoretische Physik, Universität Leipzig, Postfach 100920,04009 Leipzig, Germany

**We are thankful to Simon Mitternacht for helpful discussions regarding the peptide model and C. Dammann for peptide synthesis and purification. MB thanks the DFG (German Science

*To whom correspondence should be addressed 
Foundation) and the Wenner-Gren Foundation (Sweden) for research fellowships, and the GermanIsrael "Umbrella" program for support. $\mathrm{MB}, \mathrm{AI}$, and $\mathrm{WJ}$ are grateful for support by the GermanSwedish DAAD-STINT Personnel Exchange Programme. This work is also partially funded by the DFG under Grant Nos. JA 483/24-1/2/3, the Leipzig Graduate School of Excellence "BuildMoNa", TR 67 A4, and the German-French DFH-UFA PhD College under Grant No. CDFA-02-07. Supercomputer time at the John von Neumann Institute for Computing (NIC), Forschungszentrum Jülich, is acknowledged (Grant Nos. hlz11 and jiff39).

\begin{abstract}
The design of hybrid peptide-solid interfaces for nanotechnological applications such as biomolecular nanoarrays requires a deep understanding of the basic mechanisms of peptide binding and assembly at solid substrates. Here we show by means of experimental and computational analyses that the adsorption properties of mutated synthetic peptides at semiconductors exhibit a clear sequence-dependent adhesion specificity. Our simulations of a novel hybrid peptide-substrate model reveal the correspondence between proline mutation and binding affinity to a clean silicon substrate. After synthesizing theoretically suggested amino-acid sequences with different binding behavior, we confirm the relevance of the selective mutations upon adhesion in our subsequent atomic force microscopy experiments.
\end{abstract}

\title{
Introduction
}

In the past few years, the interest in hybrid interfaces formed by "soft" molecular matter and "hard" solid substrates has rapidly grown as such systems promise to be relatively easily accessible candidates for novel biosensors or electronic devices. The enormous progress in high-resolution microscopy and in biochemical engineering of macromolecules is the major prerequisite for studies of hybrid systems and potential applications. $\stackrel{1,2}{ }$ One particularly important problem is the selfassembly and adhesion of polymers, proteins, or protein-like synthetic peptides to solid materials such as, e.g., metals,,$\frac{3,4}{4}$ semiconductors,,$\underline{5-8}$ carbon and carbon nanotubes,,$\frac{9,10}{}$ and silica. $\underline{11,12}$ 
Peptide and substrate specific binding affinity is particularly relevant in pattern recognition processes. 13,14 Systematic experimental studies have been performed to investigate binding properties of individual amino acids in their binding behavior to selected materials. 15 Basic theoretical considerations of simplified polymer-substrate and protein-substrate models have predicted complex pseudophase diagrams. $\underline{16,17}$

In bacteriophage display experiments, only a few peptides out of a library of $10^{9}$ investigated sequences with 12 amino acid residues were found to possess a particularly strong propensity to adhere to (100) gallium-arsenide (GaAs) surfaces. $\stackrel{5}{5}$ The sequence-specificity of adsorption strength is a remarkable property, but the question remains how it is related to the individual molecular structure of the peptides. We expect that relevant mutations of sites in the amino-acid sequence can cause a change of the binding affinity. Indeed, one key aspect of our study is to show that proline is a potential candidate for switching the adsorption propensities to cleaned (100) silicon (Si) substrates.

Silicon is one of the technologically most important semiconductors, as it serves, for example, as carrier substrate in microelectronics. For this reason, electronic and surface properties of Si are well investigated. This regards, for example, oxidation processes in air $\underline{18,19}$ and water, $\underline{20,21}$ as well as the formation of hydride surface structures and the Si-binding characteristics of small organic compounds.., 22

\section{Results and Discussion}

To guide the design of peptide-silicon interfaces, we first performed extensive computer simulations of a novel hybrid model discussed below. For testing the theoretically revealed trends of adsorption propensity changes by selected mutation, we have synthesized the suggested specific mutants by means of multiple solid phase peptide synthesis. The theoretical predictions were subsequently verified in atomic-force microscope (AFM) experiments (see [figure][1][]1 and the detailed descriptions in the Supplementary Material). 
The hybrid model used in the computer simulations is composed of two parts contributing to the energy $E(\mathbf{X})$ of a peptide conformation $\mathbf{X}$ : the energy of the peptide as represented by the implicit-solvent all-atom model introduced in Refs. $\stackrel{24,25}{2}$ and the interaction of the peptide with the substrate which is modeled in a coarse-grained way. The peptide model takes into account intrinsic excluded volume repulsions between all atoms, a local potential which represents the interaction among neighboring $\mathrm{NH}$ and $\mathrm{CO}$ partial charges, hydrogen bonding energy, and the interaction between hydrophobic side chains. $\stackrel{24,25}{2}$ The substrate model consists only of atomic layers with surface specific atomic density and planar surface structure. In this simplified model, each peptide atom feels the mean field of the atomic substrate layers. The atomic density of these layers depends on the crystal orientation of the substrate at the surface. Based on these assumptions, a generic noncovalent Lennard-Jones approach for modeling the interaction between peptide atoms and surface layer is employed.9.26 We have studied this model by means of multicanonical computer simulations ${ }^{27}$ which provide us with canonical statistics for any temperature $T$. The partition function is thus given by $Z=\int \mathscr{D} \mathbf{X} e^{-E(\mathbf{X}) / R T}$, where $\mathscr{D} \mathbf{X}$ is the formal functional integral measure for all possible conformations $\mathbf{X}$ in the space of the degrees of freedom. The statistical average of any quantity $O$ is $\langle O\rangle=Z^{-1} \int \mathscr{D} \mathbf{X} O(\mathbf{X}) e^{-E(\mathbf{X}) / R T}$. In our simulations, the integral is estimated by an average over a large set of conformations (in each run about $10^{9}$ updates were performed) selected by multicanonical importance sampling. The precise modeling of the hybrid system and the multicanonical simulation methodology are described in the Supplementary Material.

The peptide with the amino acid sequence S1 [see [figure][2][]2(a)] is a good example for the substrate specificity of adsorption. In recent comparative adsorption experiments, it could be shown that although $\mathrm{S} 1$ binds strongly to $\mathrm{GaAs}(100)$, binding to $\mathrm{Si}(100)$ is very weak. ${ }^{7.8} \mathrm{In}$ contrast, the adhesion is strongly increased, if the Si substrate is oxidized. This can clearly be seen in [figure][2][]2(a), where AFM images of S1 adhered at a de-oxidized (left) and an oxidized $\mathrm{Si}(100)$ substrate (right) are shown. Peptide covered regions appear bright. A quantitative measure for the binding propensity is the peptide adhesion coefficient (PAC), which is the relative area of the surface covered by peptide clusters..$\underline{7.8}$ These PAC values are determined here by means of a cluster 
analysis of the respective AFM images. To reduce the dependence on the peptide concentration in solution, we here introduce the calibrated PAC (cPAC) as the ratio of PACs measured for the binding of the peptides to $\mathrm{Si}(100)$ and $\mathrm{GaAs}(100)$ substrates under identical conditions. GaAs(100) is chosen as a reference substrate, since the peptides considered here bind comparatively well to this substrate. The cPAC charts for S1 in [figure][2][]2 b) clearly indicate the difference of binding affinity at cleaned and oxidized substrates.

This is in contrast to sequence S3 [for sequence and AFM images see [figure][2][]2,(c)], which is a random permutation of S1 with unchanged amino acid content. Surprisingly, the binding propensity of $\mathrm{S} 3$ to $\mathrm{Si}(100)$ was found to be much larger than that of $\mathrm{S} 1 .^{\underline{7}}$ In this case, the binding affinities at cleaned and oxidized $\mathrm{Si}(100)$ substrates are similarly strong, as the cPAC charts for S3 in $[$ figure][2][]2 (b) show. In recent computational analyses of the solvent properties of these peptides, we have shown that also the folding behaviors in solution exhibit noticeable differences. This is also true at room temperature, where in both cases the population of the structurally different native folds is rather small. 23

Another remarkable result of this former computational study is that the qualitative folding behaviors of S1 and S3 are related to each other, if these sequences are pairwisely mutated at the position of proline, which occurs once in the sequences S1 and S3. $\underline{23}^{2}$ The mutated sequence S1' differs from $\mathrm{S} 1$ only by the exchange of proline $(\mathrm{P})$ at position 4 and threonine $(\mathrm{T})$ at 9 . Similarly, in S3', proline at 9 is exchanged with the aspartic acid (D) at 4, compared to S3 [see [figure][3][]3.(a)]. These replacements were reasoned by the guess that the particular steric properties of proline, and thus its place in the sequence, influence the folding. It turned out that the folding behavior of S1' in solution is close to that of S3, whereas S3' behaves rather like S1. $\stackrel{23}{ }$ Before we can address the question whether these results are also of essence for the adsorption behavior to $\mathrm{Si}(100)$, we need to discuss microscopic properties of de-oxidized $\mathrm{Si}(100)$ substrates.

In our experiments, the $\mathrm{Si}(100)$ surfaces were first cleaned in a solution of ammonium fluoride $\left(\mathrm{NH}_{4} \mathrm{~F}\right)$ and hydrofluoric acid $(\mathrm{HF}) . \underline{7}, \underline{8}$ Then, the peptide adsorption process took place in deionized water. This standard procedure ensures that the Si surface is virtually free of oxide and 
possesses strongly hydrophobic properties $\frac{18,20}{2}$ (for sample preparation and experimental details see the Supplementary Material). The initial $\mathrm{Si}-\mathrm{F}$ bonds after etching are replaced by $\mathrm{Si}-\mathrm{H}$ bonds in the rinsing process in de-ionized water. After drying the sample, AFM scans of the surface were performed. Although the oxidation also proceeds in water, $\underline{20,21}$ there are clear indications (maximum water droplet contact angle after removing the samples off the peptide solution) that the hydrophobicity of the Si samples remains largely intact during the peptide adsorption process. It is also known that Si surfaces are comparatively rough after HF treatment. $\underline{\underline{22}}$ Thus, the reactivity of the surface is influenced by steps, which depend on the offcut and its directions. This renders an atomistic modeling intricate, even more as $\mathrm{Si}(100)-2 \times 1$ surfaces are also known to form $\mathrm{Si}-$ $\mathrm{Si}$ dimers on top of the surface ${ }^{\frac{6}{}}$ with highly reactive dangling bonds. From the considerations and the experimental preparations described above, it seems plausible that these bonds are mainly passivated by hydrogen, forming hydride layers. $\underline{6} \underline{20.22}$ It should be emphasized that under these conditions the surface structure of $\mathrm{Si}(100)$ is substantially different from oxidized $\mathrm{Si}(100)$ which is polar and in effect hydrophilic. $\underline{18}$ An important result of [figure][2][]2] is that the binding of S1 and $\mathrm{S} 3$ to oxidized $\mathrm{GaAs}(100)$ and $\mathrm{Si}(100)$ surfaces is virtually independent of the substrate type (cPAC $\approx 1$ ). Thus, the top oxygen layer screens the substrate from the peptide. The different adhesion propensities to the clean (hydrated) substrates [see also [figure][2][]2] lead to the conclusion that oxidation has not yet strongly progressed during the peptide adsorption process. We conclude that the key role of water is the slowing down of the oxidation process of the $\mathrm{Si}(100)$ surface, but for the actual binding process its influence is rather small. In particular, we do not expect that stable water layers form between adsorbate and substrate.

These characteristic properties of HF treated $\mathrm{Si}(100)$ surfaces in de-ionized water effectively enter into the definition of our hybrid model of the peptide-silicon interface (for details see the "Model and Methods" section in the Supplementary Materials) which serves as the basis for our theoretical analysis and interpretation of the specificity of peptide adhesion on these interfaces.

In order to quantify the degree of adsorption, we define the ratio of heavy (non-hydrogen) atoms located in a distance $z_{i} \leq 5 \AA$ from the substrate, $n_{h}$, and the total number of heavy atoms, 
$N_{h}$, as the adsorption parameter $q=n_{h} / N_{h}$. The temperature dependence of its relative change by proline mutation, $\Delta q\left(\mathrm{~S} n \rightarrow \mathrm{S} n^{\prime}\right)=\left(\left\langle q\left(\mathrm{~S} n^{\prime}\right)\right\rangle-\langle q(\mathrm{~S} n)\rangle\right) /\langle q(\mathrm{~S} n)\rangle$ (with $\left.n=1,3\right)$, is shown in [figure][3][]3(b). The main result is that due to this selective mutation, the $\mathrm{Si}(100)$ adsorption affinity from $\mathrm{S} 1$ to $\mathrm{S} 1^{\prime}$ increases $\left[\Delta q\left(\mathrm{~S} 1 \rightarrow \mathrm{S}^{\prime}\right) \approx 0.11\right.$ at $\left.T=300 \mathrm{~K}\right]$, while it decreases by about the same amount as $\mathrm{S} 3$ is mutated to $\mathrm{S}^{\prime}{ }^{\prime}\left[\Delta q\left(\mathrm{~S} 3 \rightarrow \mathrm{S}^{\prime}\right) \approx-0.15\right.$ at $\left.T=300 \mathrm{~K}\right]$. This result is directly connected with the tendency to form secondary structures. In [figure][3][]3 (c), the respective $\alpha$-helix content [ratio of the dihedral Ramachandran angles of the inner 10 residues satisfying $\phi \in\left(-90^{\circ},-30^{\circ}\right)$ and $\left.\psi \in\left(-77^{\circ},-17^{\circ}\right)\right]$ and $\beta$-strand content [dihedral angles in the intervals $\phi \in\left(-150^{\circ},-90^{\circ}\right)$ and $\left.\psi \in\left(90^{\circ}, 150^{\circ}\right)\right]$ of the bound conformations are shown. We define a conformation as bound to the substrate, if at least $2 \%$ of the heavy atoms are within a 5 $\AA$ distance from the surface. There is a clear tendency that residues of S1 and S3' are rather in $\alpha$ state and residues of $\mathrm{S} 3$ and $\mathrm{S} 1$ ' in $\beta$ state. Yet the small secondary-structure contents are quite similar to what we found for the peptides in solution (without substrate), $\underline{23}$ which were qualitatively consistent with analyses of CD spectra. $\stackrel{-}{-}$ It is a noticeable result that here secondary structures are not stabilized near the cleaned $\mathrm{Si}(100)$ substrate, whereas in recent adsorption experiments of a synthetic peptide binding at silica nanoparticles, a stabilization of $\alpha$-helices was observed. $\underline{\underline{12}}$

The experimental results shown in [figure][3][3](d) confirm that the proline mutation of S1 indeed increases the $\mathrm{Si}(100)$ binding affinity, while an analogous mutation decreases the binding strength of $\mathrm{S} 3$ by about the same value. The AFM images indicate the increased substrate coverage for S1' and the decrease in the case of S3' [cp. with the corresponding images in [figure][2][]2 a) and (c), respectively]. By measuring the associated cPAC values, we find that $\Delta \mathrm{cPAC}\left(\mathrm{S} 1 \rightarrow \mathrm{S} 1^{\prime}\right)=$ $\operatorname{cPAC}\left(\mathrm{S}^{\prime}\right)-\operatorname{cPAC}(\mathrm{S} 1) \approx 0.27$ and $\Delta \mathrm{cPAC}\left(\mathrm{S} 3 \rightarrow \mathrm{S} 3^{\prime}\right) \approx-0.25$. This convincingly confirms our theoretical prediction from the hybrid-model simulations. 


\section{Conclusions}

In summary, we have predicted by computer simulations and verified by AFM experiments that a selected proline mutation of short peptides facing a de-oxidized silicon substrate can substantially change the binding affinity in a very predictive and specific way. We could also show that this behavior is in part due to a qualitatively different folding behavior of the mutated sequences in the vicinity of the substrate. The proline position most likely also affects the aggregation properties $\underline{8}$ of the peptides and thereby indirectly again their binding characteristics. Building up on simulations of single-molecule behavior, as those discussed in the present manuscript, simulating coupled folding and aggregation while binding will therefore constitute a rewarding future project. Gaining deeper insights into the general principles of binding specificities is a first fundamental step towards the design of nanosensors with specific biomedical applications. Thus, the extension of our study to biomolecules is natural and the identification of unique bioprotein adsorption signals in experiments with nanoarrays of several materials is a prerequisite for future applicability of such hybrid systems in biotechnology.

The precise modeling of the hybrid system, the multicanonical simulation methodology and details of the peptide selection, the AFM experiments and the sample preparation are described in the Supplementary Material.

\section{References}

(1) M. Sarikaya, C. Tamerler, A. K.-Y. Jen, K. Schulten, F. Baneyx, Nature Mat. 2003, 2, 577585.

(2) J. J. Gray, Curr. Opin. Struct. Biol. 2004, 14, 110-115.

(3) S. Brown, Nature Biotechnol. 1997, 15, 269-272.

(4) L. Delle Site, C. F. Abrams, A. Alavi, K. Kremer, Phys. Rev. Lett. 2002 ,89, 156103-1-4. 
(5) S. R. Whaley, D. S. English, E. L. Hu, P. F. Barbara, A. M. Belcher, Nature 2000, 405, $665-668$.

(6) J. M. Buriak, Chem. Rev. 2002, 102, 1271-1308.

(7) K. Goede, P. Busch, M. Grundmann, Nano Lett. 2004, 4, 2115-2120.

(8) K. Goede, M. Grundmann, K. Holland-Nell, A. G. Beck-Sickinger, Langmuir 2006, 22, 8104-8108.

(9) R. Hentschke, Macromol. Theory Simul. 1997, 6, 287-316.

(10) S. Wang, E. S. Humphreys, S.-Y. Chung, D. F. Delduco, S. R. Lustig, H. Wang, K. N. Parker, N. W. Rizzo, S. Subramoney, Y.-M. Chiang, A. Jagota, Nature Mat. 2003, 2, 196-200.

(11) H. Heinz, H. Koerner, K. L. Anderson, R. A. Vaia, B. L. Farmer, Chem. Mat. 2005, 17, $5658-5669$.

(12) M. Lundqvist, P. Nygren, B.-H. Jonsson, K. Broo, Angew. Chem. Int. Ed. 2006, 45, 81698173.

(13) A. J. Golumbfskie, V. S. Pande, A. K. Chakraborty, Proc. Natl. Acad. Sci. USA 1999, 96, 11707-11712.

(14) T. Bogner, A. Degenhard, F. Schmid, Phys. Rev. Lett. 2004, 93, 268108-1-4.

(15) R. L. Willett, K. W. Baldwin, K. W. West, L. N. Pfeiffer, Proc. Natl. Acad. Sci. USA 2005, $102,7817-7822$.

(16) M. Bachmann, W. Janke, Phys. Rev. Lett. 2005, 95, 058102-1-4.

(17) M. Bachmann, W. Janke, Phys. Rev. E 2006, 73, 020901(R)-1-4.

(18) R. G. Frieser, J. Electrochem. Soc. 1974, 121, 669-672.

(19) A. Hemeryck, N. Richard, A. Estève, M. Djafari Rouhani, Surf. Sci. 2007, 601, 2339-2343. 
(20) L. Ling, S. Kuwabara, T. Abe, F. Shimura, J. Appl. Phys. 1993, 73, 3018-3022.

(21) M. K. Weldon, B. B. Stefanov, K. Raghavachari, Y. J. Chabal, Phys. Rev. Lett. 1997, 79, 2851-2854.

(22) Y. J. Chabal, K. Raghavachari, Phys. Rev. Lett. 1984, 53, 282-285.

(23) S. Mitternacht, S. Schnabel, M. Bachmann, W. Janke, A. Irbäck, J. Phys. Chem. B 2007, 111, 4355-4360.

(24) A. Irbäck, S. Mohanty, Biophys. J. 2005, 88, 1560-1569.

(25) A. Irbäck, S. Mohanty, J. Comput. Chem. 2006, 27, 1548-1555.

(26) W. A. Steele, Surf. Sci. 1973, 36, 317-352.

(27) B. A. Berg , T. Neuhaus Phys. Rev. Lett. 1992, 68, 9-12. 


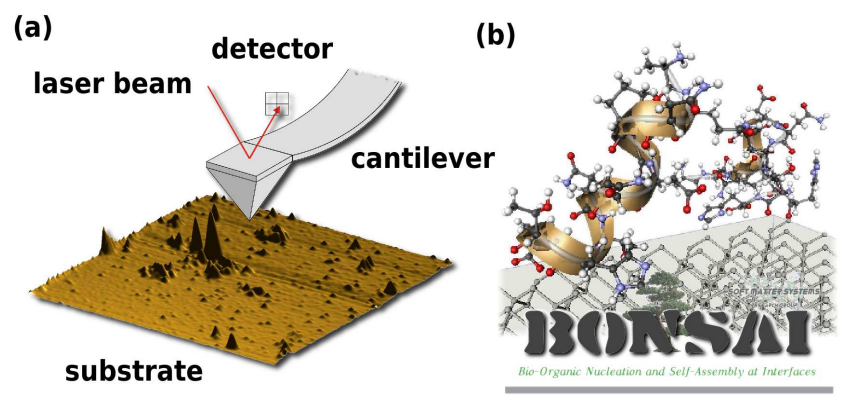

Figure 1: Methodologies. (a) Sketch of the atomic-force microscope. The original AFM image exhibits S1 peptide clusters on an oxidized $10 \times 10 \mu \mathrm{m}^{2}$ silicon substrate. The height of the highest cluster is $56 \mathrm{~nm}$. (b) Computer simulations were performed with the BONSAI package that we developed for Monte Carlo simulations of peptide models. The snapshot shows S1 peptides forming helical segments near a silicon substrate. 
(a) $\mathrm{S} 1$
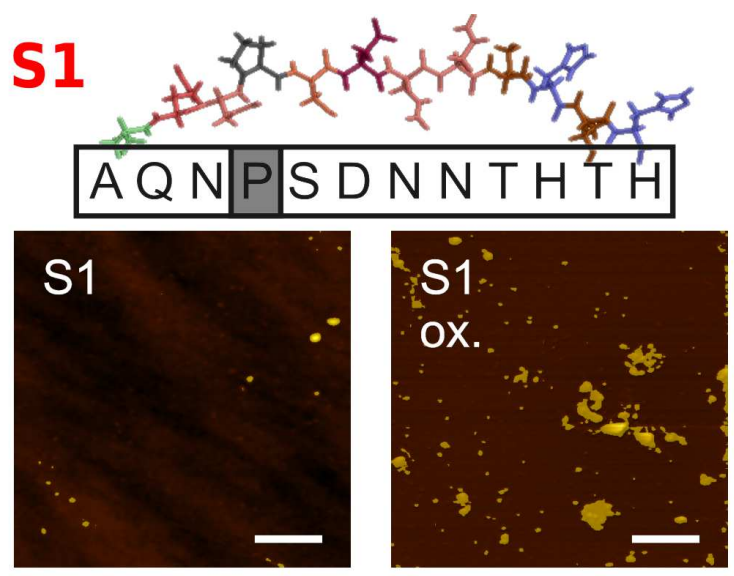

(b)

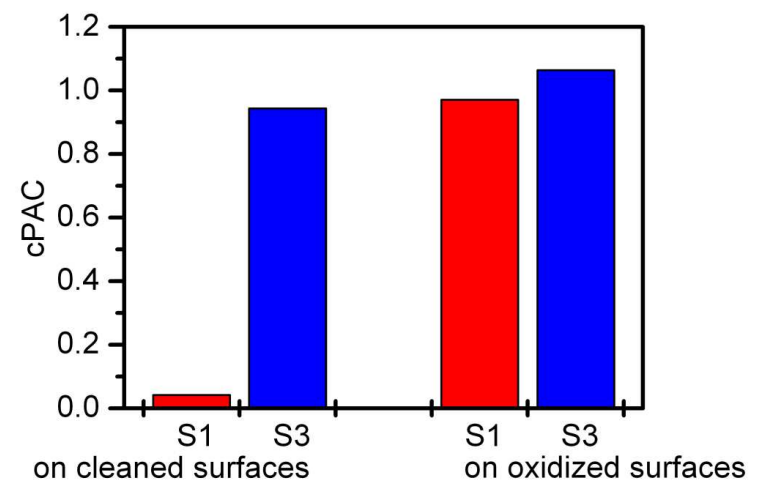

(c)

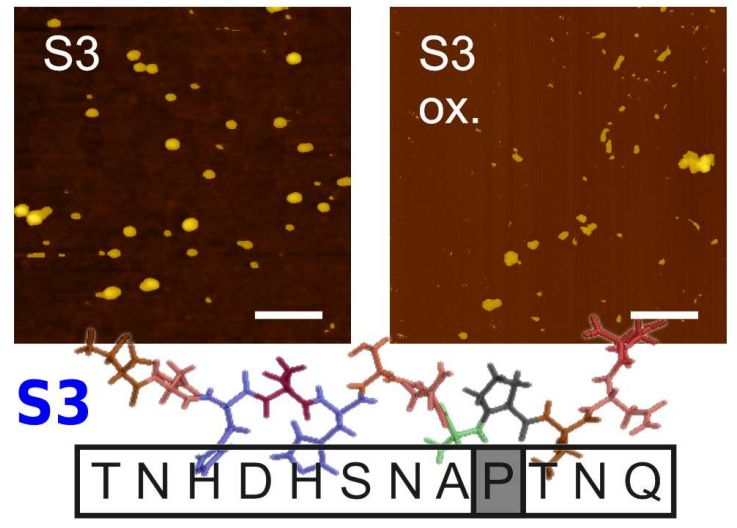

Figure 2: Adsorption to cleaned and oxidized substrates. Exemplified AFM images of peptides (a) S1 and (c) S3 adsorbed to cleaned (de-oxidized) and oxidized Si(100) surfaces. The AFM scale bar is $1 \mu \mathrm{m}$. (b) Calibrated peptide adhesion coefficients (cPAC) for S1 and S3 adsorption to cleaned and oxidized $\mathrm{Si}(100)$ substrates. Single-letter code of amino acids occurring in the peptides of our study: A - alanine, $\mathrm{D}$ - aspartic acid, $\mathrm{H}$ - histidine, $\mathrm{N}$ - asparagine, $\mathrm{P}$ - proline, $\mathrm{Q}$ - glutamine, $\mathrm{S}$ - serine, $\mathrm{T}$ - threonine. 
(a)
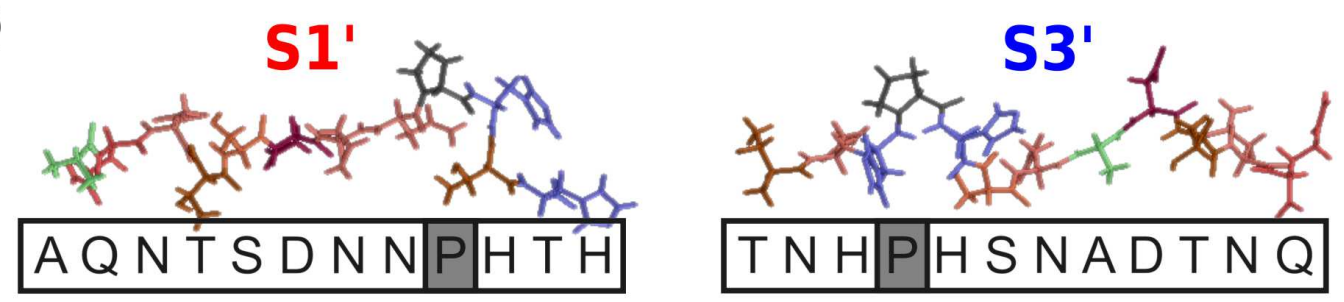

(b)

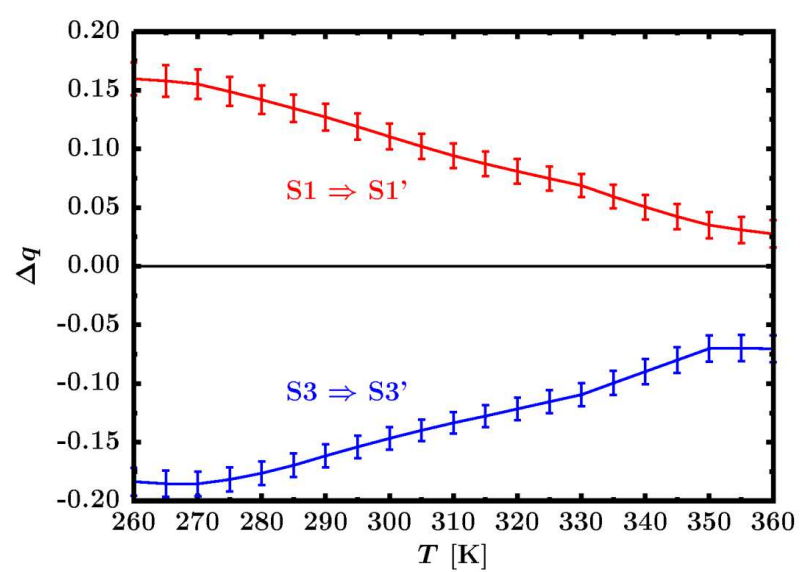

(c)

(d)
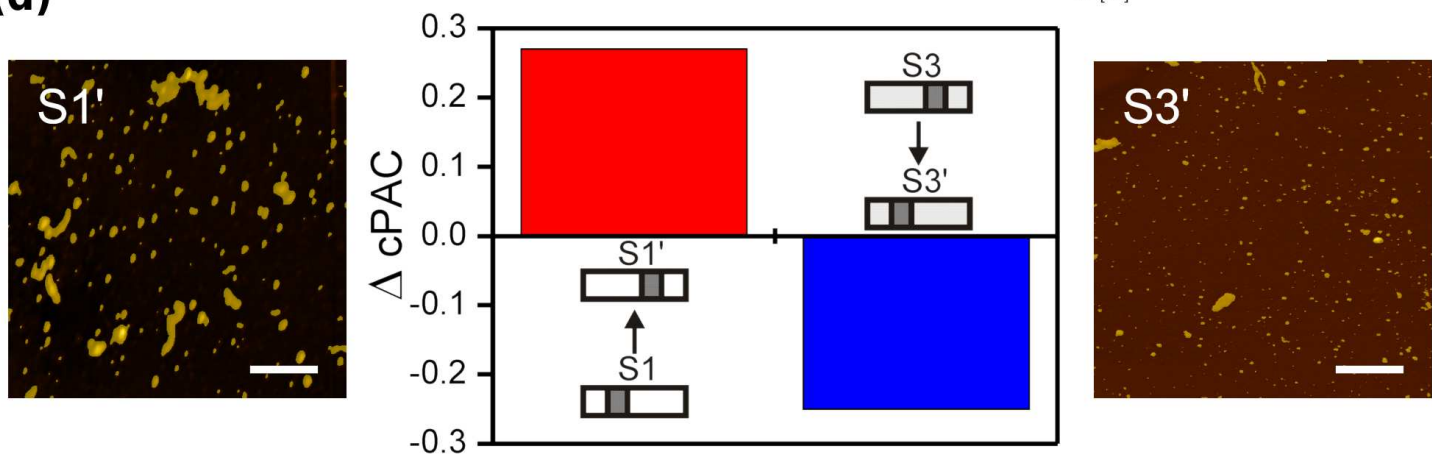

Figure 3: Reversed adsorption propensity of proline-mutated peptides. (a) Proline-mutated sequences S1' and S3'. (b) Adsorption parameter $\Delta q$ as a function of temperature from our computer simulations. The prediction is that the proline mutation of $\mathrm{S} 1$ causes an increase of binding affinity, whereas mutating S3 leads to a decrease, i.e., proline mutation reverses the binding propensity of S1 and S3. (c) $\alpha$-helix content $\left\langle n_{\alpha}\right\rangle_{\mathrm{b}}$ and $\beta$-strand content $\left\langle n_{\beta}\right\rangle_{\mathrm{b}}$ of bound conformations also exhibit a pairwise reversal of the tendency to form secondary structures. Exemplified conformations depicted in the insets are lowest-energy structures identified in the simulations (with rather small populations at room temperature) and represent the preferred trends in secondary-structure formation: helical for S1 and S3', sheet-like for S1' and S3. (d) Confirmation by AFM experiments at room temperature. The cPAC increases by proline-mutating $\mathrm{S} 1$ and decreases by about the same value if $\mathrm{S} 3$ is mutated. AFM scale bar $1 \mu \mathrm{m}$. 


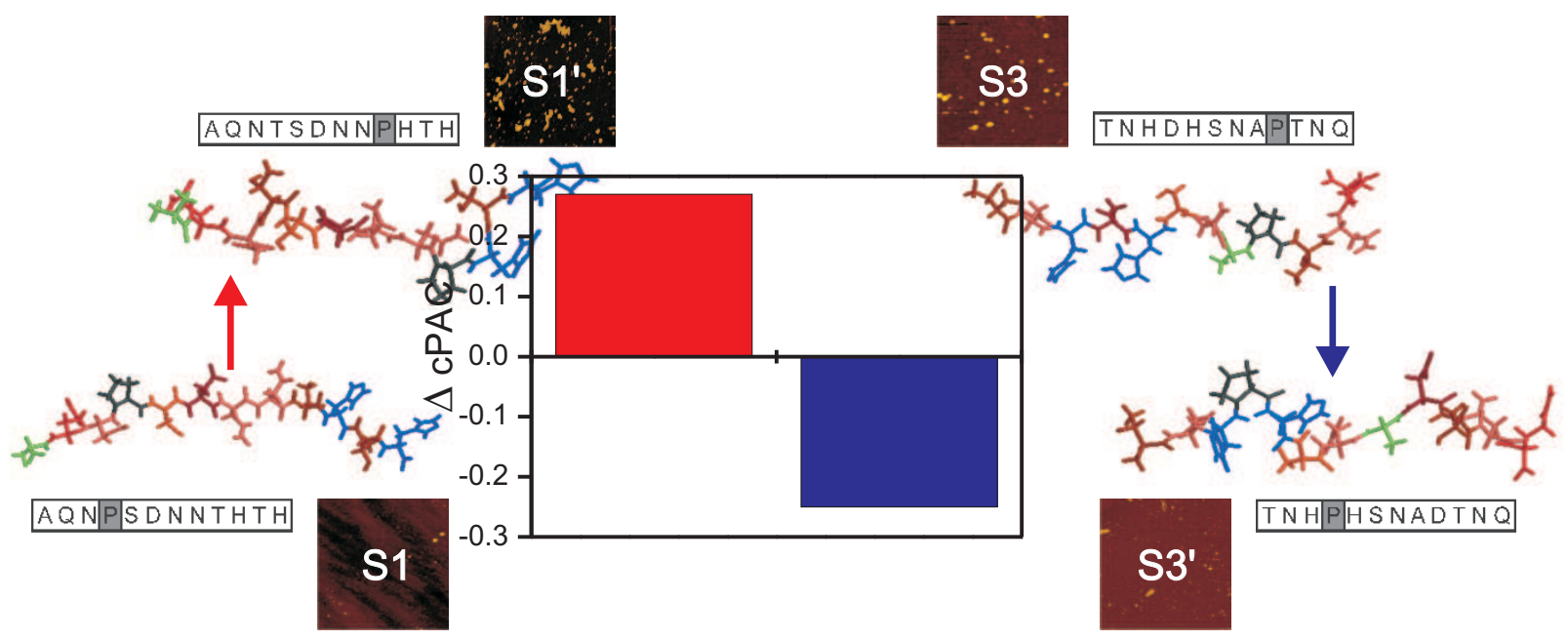

Figure 4: Suggested TOC graphic. Designing novel hybrid peptide-solid interfaces for nanotechnology requires a sound understanding of peptide binding and assembly at substrates. Here we show by means of experimental and computational analyses for a novel hybrid peptide-substrate system that the adsorption properties of mutated synthetic peptides at semiconductors exhibit a clear sequence-dependent adhesion specificity, which is mainly governed by the positions of crucial amino acids. 